\title{
Raman Characterisation of $\mathrm{Cd}_{1-x} \mathrm{Zn}_{x}$ Te Thick Polycrystalline Films Obtained by the Close-Spaced Sublimation
}

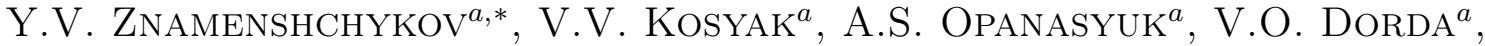 \\ P.M. FOCHUK ${ }^{b}$ AND A. MEDVIDS ${ }^{c}$ \\ ${ }^{a}$ Sumy State University, 2, Rymsky Korsakov Str., 40007 Sumy, Ukraine \\ ${ }^{b}$ Chernivtsi National University, 2, Kotsiubynskogo Str., Chernivtsi, Ukraine \\ ${ }^{c}$ Riga Technical University, 3 Paula Valdena Str., LV-1048, Riga, Latvia \\ (Received May 8, 2017; in final form September 12, 2017)
}

\begin{abstract}
In this work, we studied the Raman spectra of thick polycrystalline $\mathrm{Cd}_{1-x} \mathrm{Zn}_{x} \mathrm{Te}$ (CZT) films with $x$ ranged from 0.06 to 0.68 . Additionally, the surface morphology and structural properties were studied in order to determine the crystalline quality of the samples. The Raman spectra had a two-mode behavior typical for CZT solid solution and showed CdTe- and ZnTe-like longitudinal and transverse optical modes. The relationship between the frequencies of CdTe- and ZnTe-related modes on $x$ was studied. We observed the deviation of the compositional dependence of phonon mode frequencies for polycrystalline CZT films in comparison with a similar dependence for CZT single crystals. Such deviation was caused by the effect of structural defects in polycrystalline films on frequencies of vibrational modes. The values of excitation wavelength, which allow achieving of high signal-to-noise ratio on the Raman spectra of CZT films with different zinc concentration in the result of resonant enhancement of phonon modes intensities, were experimentally determined.
\end{abstract}

DOI: 10.12693/APhysPolA.132.1430

PACS/topics: 81.15.Ef, 81.05.Dz

\section{Introduction}

Due to high resistivity, high atomic number, tunable band gap in the range from $1.50(\mathrm{CdTe})$ to $2.26 \mathrm{eV}(\mathrm{ZnTe})$ depending on Zn concentration [1], $\mathrm{Cd}_{1-x} \mathrm{Zn}_{x} \mathrm{Te}(\mathrm{CZT})$ ternary semiconductor is widely used as a material for radiation detectors $[2,3]$, photodetectors, tandem solar cells [4]. In particular, thick polycrystalline CZT films are promising material for fabrication of low-cost large-area radiation detectors [5]. Wherein the application of CZT as a detector requires the material with high crystalline quality, uniform components distribution and free of secondary phases, which can affect the detector performance. Various techniques are used for deposition of CZT films, such as magnetron sputtering [6], metal-organic chemical vapor deposition [7], electrodeposition [8], molecular-beam epitaxy [9], thermal vacuum evaporation [10], closed-space vacuum sublimation [11]. Closed-space vacuum sublimation (CSVS) is a promising low-cost technique, which allows to obtain high-quality CZT films with controllable Zn concentration [12].

X-ray diffraction is widely used method, which provides information about phase composition and crystalline quality of the material $[13,14]$ and allows to estimate $\mathrm{Zn}$ concentration in CZT. The crystalline quality of the material is determined using full width at half maximum (FWHM) of diffraction peaks. The phase analysis

\footnotetext{
* corresponding author; e-mail: yaroslav. znamenshchykov@gmail.com
}

of the material is carried out by matching of diffraction peaks position with the reference data. Zn concentration in CZT solid solution can be determined from XRD data using the Vegard law, which describes the linear dependence of $\mathrm{Cd}_{1-x} \mathrm{Zn}_{x}$ Te lattice constant on $x$.

The Raman spectroscopy is very sensitive to phase composition of materials; this method also allows to estimate crystalline quality and Zn concentration in CZT. The crystal quality of the samples can be estimated according to the presence and intensity of phonon replicas on the Raman spectra [15]. As a rule, the Raman spectra of cubic phase CZT include CdTe- and ZnTe-related modes of longitudinal (LO) and transverse (TO) optical phonons. The dependences of CdTe- and ZnTe-related mode frequencies on $x$ were investigated for CZT single crystals $[15,16]$ and epitaxial films [17]. However, the dependence of phonon modes frequencies on $x$ was not studied for polycrystalline CZT films. At the same time, microstrains and structural defects in polycrystalline films can affect the phonon modes frequencies [18, 19], therefore the detailed study of the Raman spectra properties of polycrystalline CZT films is required.

The goals of this work are: the study of structural and optical properties of CZT films; the analysis of the Raman spectra of polycrystalline CZT films with different $\mathrm{Zn}$ concentration; study of phonon modes frequencies dependence on $\mathrm{Zn}$ concentration and comparison of the obtained results for CZT polycrystalline films with the similar ones for CZT single crystals; and determination of optimal conditions, namely excitation wavelength, for measurement of Raman spectra of polycrystalline CZT films with different zinc concentration, which provide high signal-to-noise ratio in the Raman spectra. 


\section{Materials and methods}

The CZT films were deposited on ITO-coated glass substrates by CSVS method analogously to our previous works $[14,20,21]$. The mixture of CdTe and ZnTe powders was used as a source. In order to obtain CZT films with different $\mathrm{Zn}$ concentration the mass ratio $M_{R}$ of CdTe and ZnTe powders was varied from film to film as follows: 20 (CZT1), 10 (CZT2), 7 (CZT3), 5 (CZT4), 2.5 (CZT5), 2 (CZT6). The growth conditions for films were the same, namely, the substrate temperature was $400^{\circ} \mathrm{C}$ and the evaporator temperature was $700^{\circ} \mathrm{C}$.

The surface morphology of the films has been investigated by Renishaw InVia optical microscope. The thicknesses of the films were determined from the cross-section optical images.

Structural analysis was carried out by Rigaku Ultima + X-ray diffractometer, using $K_{\alpha} \mathrm{Cu}$ radiation source, the scan step was 0.0032 -theta degrees. The samples were measured in the $2 \theta$ angle range from $20^{\circ}$ to $80^{\circ}$. The peak intensities were normalized to the intensity of (111) peak of the cubic phase. Phase analysis was performed by comparison of the inter-planar distances as well as relative intensities measured from the samples with reference Joint Committee on Powder Diffraction Standards (JCPDS card No. 15-0770) data [22]. Precise values of the lattice parameter were determined by the extrapolated Nelson-Riley method [23].

Room temperature Raman spectra (RS) were studied using Renishaw InVia 90V727 micro-Raman spectrometer. Semiconductor IR $(\lambda=785 \mathrm{~nm})$, red HeNe $(\lambda=633 \mathrm{~nm})$ and green $\operatorname{Ar}(\lambda=514 \mathrm{~nm})$ lasers were used as an excitation source. Measurement parameters were the same as in previous work [12]. Registration of signal was carried out by CCD camera. Calibration of the spectrometer was done by measuring the Raman line at $520 \mathrm{~cm}^{-1}$ of the silicon wafer. The power of excitation was set as a percentage of the nominal power of 25 , $12.5,300 \mathrm{~mW}$ for the green $\mathrm{Ar}$, red $\mathrm{HeNe}$, semiconductor IR lasers, respectively. The diameter of the laser spot is dependent on wavelength of the laser radiation, and it was calculated to be $0.7,0.9$, and $1.1 \mu \mathrm{m}$ for the green, red and IR lasers, respectively. The accumulation time and power of excitation were chosen so as to obtain high signal-to-noise ratio while avoiding damage of the surface and high background luminescence.

\section{Results and discussion}

As a result of mass ratio variation of CdTe and $\mathrm{ZnTe}$ powders in initial powder, the obtained films had following values of $x: x=0.06$ (CZT1), $x=0.24$ (CZT2), $x=0.40$ (CZT3), $x=0.46$ (CZT4), $x=0.63$ (CZT5), $x=0.68$ (CZT6)

The optical images of the CZT films surface are shown in Fig. 1. The CZT1 and CZT2 samples consist of uniform grains with clearly defined boundaries and an average grain size $(D)$ of $\approx 20$ and $\approx 15 \mu \mathrm{m}$ for CZT1 and
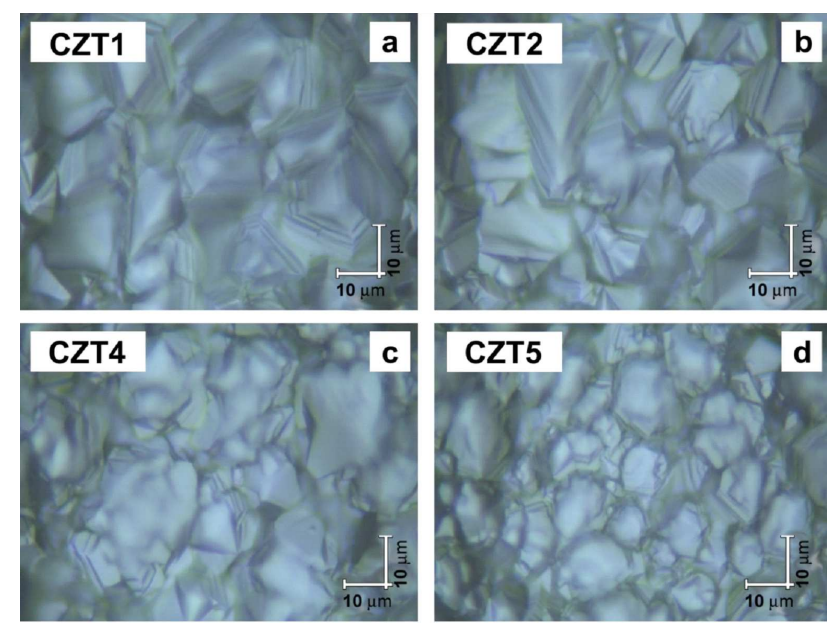

Fig. 1. Optical images of CZT samples surface: (a) $\mathrm{CZT} 1 \quad(x=0.06), \quad(\mathrm{b}) \mathrm{CZT} 2 \quad(x=0.24)$, (c) CZT4 $(x=0.46),(d) \operatorname{CZT5}(x=0.63)$.

CZT2 samples, respectively. The surface of CZT4 sample consists of non-uniform grains with $D$ ranged from 7 to $15 \mu \mathrm{m}$. The surface of CZT5 sample is more uniform in comparison with CZT4; it consists of rounded grains with an $\approx 9 \mu \mathrm{m}$ average grain size. Thus, the relationship between surface quality and $\mathrm{Zn}$ concentration shows complex behaviour. Modification of the films surface could be caused by deterioration of CZT crystal structure due to increasing amount of $\mathrm{Zn}$ atoms, as well as non-optimal growth conditions for films with high $\mathrm{Zn}$ concentration [24]. The thicknesses of the films were measured from the film cross-section and were $\approx 60 \mu \mathrm{m}$.

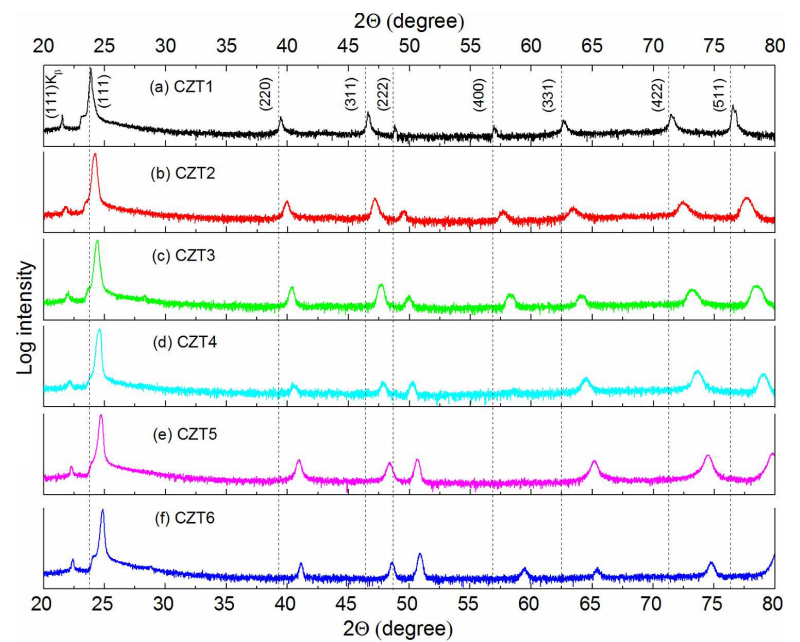

Fig. 2. XRD patterns of CZT1 $(x=0.06)$, CZT2 $(x=$ $0.24), \mathrm{CZT} 3(x=0.40), \mathrm{CZT} 4(x=0.46), \mathrm{CZT} 5(x=$ $0.63)$, CZT6 $(x=0.68)$ samples.

The XRD patterns from CZT1-CZT6 samples (Fig. 2) include peaks from $(111),(220),(311),(222),(422),(331)$ and (511) planes of the cubic (zinc-blende) phase [22]. 
With increasing of $\mathrm{Zn}$ concentration, the diffraction peaks are shifting towards higher values of diffraction angles from corresponding crystallographic planes. The dominant (111) peak corresponds to a preferential orientation in the [111] direction. The peaks of other crystal phases such as the hexagonal phase of CZT were not observed, it indicates the permanence of phase composition with increase of of $\mathrm{Zn}$ concentration.

The X-ray diffraction analysis showed that in the result of evaporation of $\mathrm{CdTe} / \mathrm{ZnTe}$ powders mixture, the CZT solid solution was formed, external compounds, such as CdTe and ZnTe, were not observed. The diffraction peaks were unsplit and had a symmetrical shape, which indicates the homogeneous distribution of $\mathrm{Zn}$ atoms in the volume of solid solution.

TABLE I

Structural and microstructural parameters of CZT films: lattice parameter (111) A, lattice parameter (NelsonRiley) $B$, CSD size $D$, in [nm], $x$, FWHM (111) $\left[{ }^{\circ}\right] C$, and $M S \times 10^{-3} E$.

\begin{tabular}{c|c|c|c|c|c|c}
\hline \hline & $A$ & $B$ & $x$ & $C$ & $D$ & $E$ \\
\hline CZT1 & 0.64468 & 0.64576 & 0.06 & 0.080 & 133 & 1.4 \\
CZT2 & 0.63592 & 0.63889 & 0.24 & 0.215 & 45 & 4.1 \\
CZT3 & 0.63013 & 0.63289 & 0.40 & 0.223 & 43 & 4.2 \\
CZT4 & 0.62579 & 0.63053 & 0.46 & 0.228 & 42 & 4.3 \\
CZT5 & 0.62362 & 0.62411 & 0.63 & 0.228 & 42 & 4.3 \\
CZT6 & 0.61972 & 0.62191 & 0.68 & 0.180 & 54 & 3.3
\end{tabular}

The values of lattice parameter were calculated from (111) peak position, as well as by the Nelson-Riley method [23] (Table I). More precise values of lattice parameter (Nelson-Riley method) were used for calculation of $x$ in $\mathrm{Cd}_{1-x} \mathrm{Zn}_{x}$ Te samples by the Vegard law $[25,26]$. These results are presented in Table I.

The microstructural parameters (coherent scattering domains (CSD) size and microstrains) were calculated by the method, described in Refs. [27, 28], using FWHM of (111) diffraction peak (Table I). Analogously to [25, 29], the dependence of FWHM peak (111) and therefore the dependence of CSD size and microstrains level on $x$ had a nonlinear behavior; in particular CZT4 and CZT5 samples, with $x=0.46$ and $x=0.63$ respectively, showed the maximal value of FWHM of (111) peak. This effect is explained by the following process. The incorporation of $\mathrm{Zn}$ atoms into the CdTe crystal lattice leads to its deformation and structural defects, since atomic radius of zinc atom $(142 \mathrm{pm})$ is smaller than cadmium (161 pm). Wherein, as it was shown in [25, 30], the maximal microdeformation level in CZT solid solution is reached at $x=0.5$.

As it is known from [15, 17, 31], the Raman spectra of CZT solid solution show the two-mode behavior of lattice vibrations. As a rule, the Raman spectra of CZT include CdTe- and ZnTe-like LO and TO optical phonon modes [15-17]. According to [1, 15-17, 31], CdTe- and ZnTe-like modes in CZT Raman spectra are shifted from their position in pure compounds depending on $\mathrm{Zn}$ concentration. In particular, with increase of $\mathrm{Zn}$ concentration, the frequencies of $\mathrm{TO}_{1}(\mathrm{CdTe}), \mathrm{LO}_{2}(\mathrm{ZnTe})$, $\mathrm{TO}_{2}(\mathrm{ZnTe})$ modes are increasing, while the frequency of $\mathrm{LO}_{1}(\mathrm{CdTe})$ mode is decreasing. Figure 3 shows the dependences of CdTe- and ZnTe-related modes on $x$, which were obtained in [16] for CZT single crystals. These dependences were used as a reference for peak identification in the Raman spectra of CZT films.

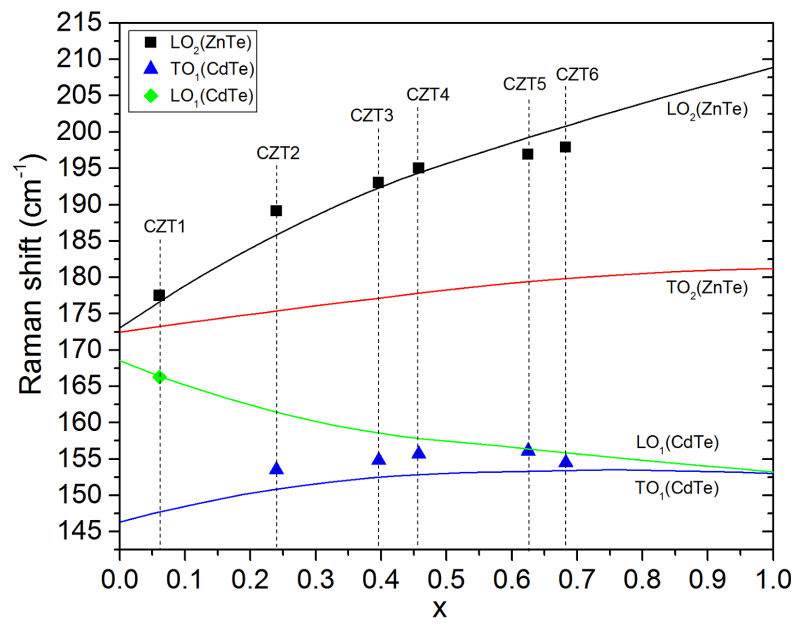

Fig. 3. The compositional dependences of frequencies of CdTe and ZnTe-like modes. Solid lines - reference data [16], dots - experimental results (measured with 785 (CZT1) and $633 \mathrm{~nm}$ (CZT2-CZT6) excitation).

The presence of Te-inclusions [31, 32], which usually occur in the process of CZT single crystal growth, leads to the appearance of Te-related modes in the Raman spectra of CZT. The appearance of the strong Te-related modes could be caused by local overheating followed by Te-enrichment of the surface, as a result of the application of high energy green excitation for the Raman study $[33,34]$.

In order to avoid the surface overheating, as well as to obtain the Raman spectra with high signal-to-noise ratio, suitable for reliable analysis, it was important to choose the optimal accumulation time, excitation power and wavelength for the Raman measurements. However, the presence of numerous crystalline defects in polycrystalline CZT films makes it difficult to obtain the Raman spectra with high signal-to-noise ratio. As it was determined in $[17,31,35]$, the high signal-to-noise ratio of the Raman spectra was achieved under resonant conditions, when the energy of incoming photons $E_{i n}$ was close to the material band gap $E_{0}$. In particular, in [17,31], $E_{\text {in }}$ was chosen slightly above the band gap of the material. Since band gap of CZT increases with $x$, it is necessary to choose optimal excitation wavelength for films with $x$, ranged from 0 to 1 , in order to achieve resonant enhancement of the Raman spectra. In Ref. [12] we determined that application of infrared laser $(E=1.579 \mathrm{eV})$ is suitable for the Raman spectra measurement of Zn-poor films $(x<0.10)$. At the same time, application of the red laser 
$(E=1.958 \mathrm{eV})$ is more preferable for films with $x$ ranged from 0.10 to 0.60 . However, for CZT films with $x>0.60$ the determination of the optimal measurement parameters was not carried out. Apart from that, in Ref. [31] the Raman spectra of CZT films with $x=0.80$ and $x=1$ were measured using the green laser $(E=2.41 \mathrm{eV})$.

Therefore, in the present work, the infrared laser was applied to measure CZT1 sample Raman spectrum. The spectra of CZT1-CZT6 samples were measured using the red laser. The green laser was applied to measure Raman spectra of Zn-rich CZT5 and CZT6 samples.

Figure 4 shows the room-temperature Raman spectra of CZT films, measured at 785 (CZT1) and $633 \mathrm{~nm}$ (CZT2-CZT6) laser excitation. The spectrum of CZT1 sample was not presented in Fig. 4 because of high background luminescence, which did not allow to identify vibrational modes.

The Raman spectra of all investigated samples, analogously to $[15,17,31]$, show two-mode behaviour. Taking into account that the frequency of the most intensive peak on the spectra increases with $x$ (from 177 (CZT1) to $197 \mathrm{~cm}^{-1}$ (CZT6)), this peak was assigned with $\mathrm{LO}_{2}(\mathrm{ZnTe})$ mode (Fig. 3). Since frequency of the peak at $\approx 155 \mathrm{~cm}^{-1}$ in the spectra of CZT2-CZT6 samples weakly depends on $x$, analogously to $[15,17]$, this peak was attributed to $\mathrm{TO}_{1}(\mathrm{CdTe})$ mode. The shallow peak at $166 \mathrm{~cm}^{-1}$ on the spectrum of CZT1, according to Fig. 3, could be assigned with $\mathrm{LO}_{1}(\mathrm{CdTe})$ mode.

As it is seen from Fig. 3, there is some discrepancy between frequencies of phonon modes for CZT films and reference data, namely obtained values of $\mathrm{TO}_{1}(\mathrm{CdTe})$ mode frequency are approximately $3 \mathrm{~cm}^{-1}$ higher than reference data, and the deviation of $\mathrm{LO}_{2}(\mathrm{ZnTe})$ mode frequency from reference data for CZT2, CZT5, CZT6 samples is about $3 \mathrm{~cm}^{-1}$. The deviation of values of phonon modes frequencies for polycrystalline CZT films from reference data [16], obtained for CZT single crystals, could be caused by microstrains of the crystal lattice, analogously to $[18,19]$, where materials with zinc-blende structure were investigated. However, in general, the obtained dependence is well correlated with the commonly accepted theory of optical phonons in CZT solid solution.

The Raman spectra from CZT films include resonant overtones of phonon modes. The spectrum of CZT1 sample includes peaks of the second-order $2 \mathrm{LO}_{1}(\mathrm{CdTe})$ and $2 \mathrm{LO}_{2}(\mathrm{ZnTe})$ modes, the spectra of CZT2-CZT5 samples include peaks of $2 \mathrm{TO}_{1}(\mathrm{CdTe})$ and $2 \mathrm{LO}_{2}(\mathrm{ZnTe})$ modes. Wherein, analogously to the first-order $\mathrm{TO}_{1}(\mathrm{CdTe})$ and $\mathrm{LO}_{2}(\mathrm{ZnTe})$ modes, the frequency of $2 \mathrm{TO}_{1}(\mathrm{CdTe})$ weakly depends on $x$, and the frequency of $2 \mathrm{LO}_{2}(\mathrm{ZnTe})$ increases with $x$, respectively. Peaks at $\approx 575 \mathrm{~cm}^{-1}$ in the spectra of CZT3 and CZT4 samples, as well as the weak peak at $\approx 570 \mathrm{~cm}^{-1}$ in the spectrum of CZT2 sample, were assigned to the third-order resonant overtones $3 \mathrm{LO}_{2}(\mathrm{ZnTe})$.

The Raman spectra from CZT5 and CZT6 samples, measured with green laser excitation $(\lambda=514 \mathrm{~nm}$, $E=2.41 \mathrm{eV})$, are presented in Fig. 5. The spectrum of CZT5 sample includes the peak of $\mathrm{LO}_{2}(\mathrm{ZnTe})$ mode
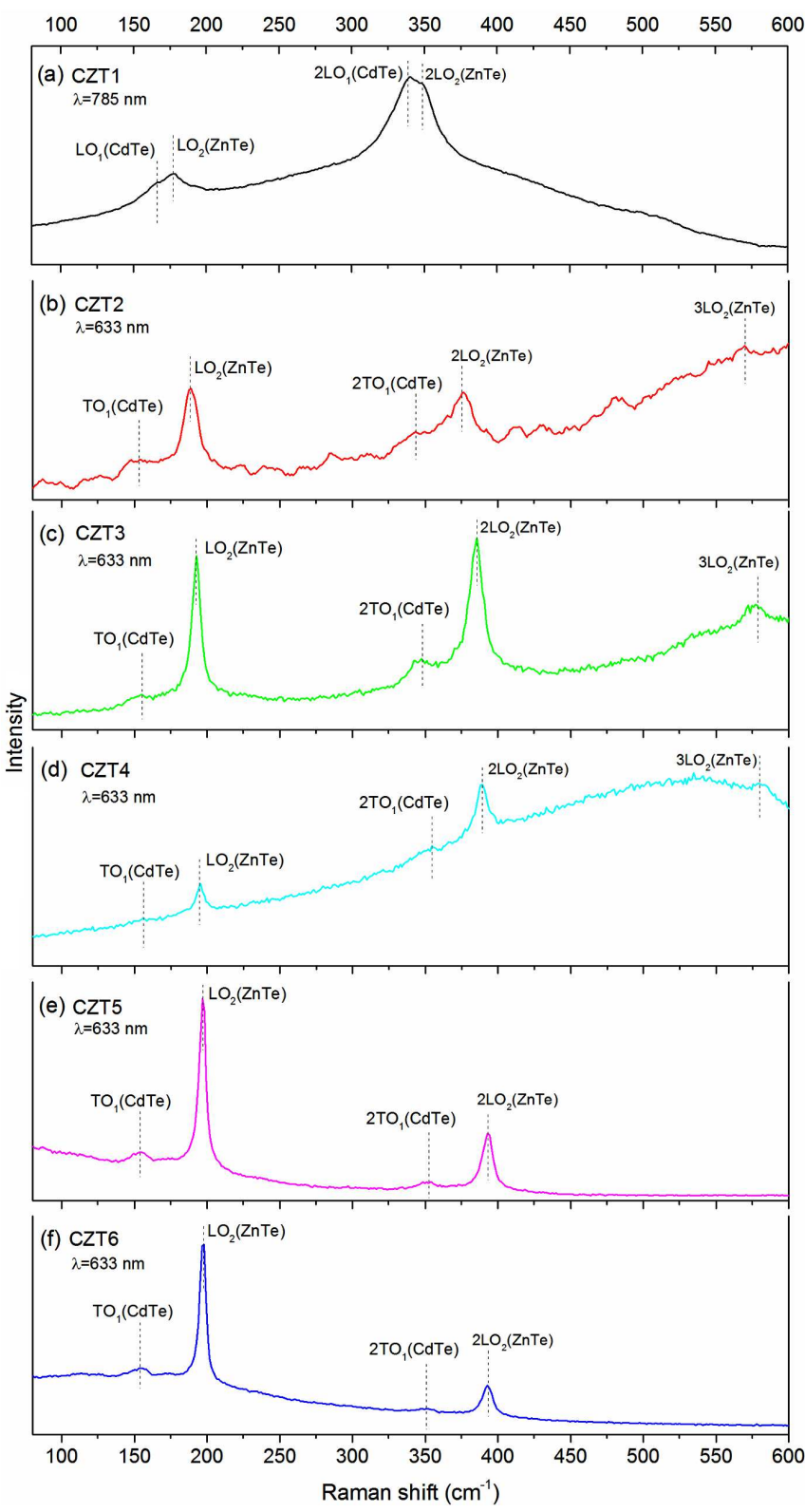

Fig. 4. Room temperature Raman spectra of the CZT films, measured with 785 (CZT1) and $633 \mathrm{~nm}$ (CZT2CZT6) excitation.

and its second- and third-order resonant overtones. The strong $A_{1}(\mathrm{Te})$ and $E_{T O}(\mathrm{Te})$ modes were observed in the spectrum of CZT5 sample. The appearance of Te-related modes could be caused by local overheating of the surface due to using of green excitation with high energy and, therefore, Te-enrichment of the surface [12, 33, 34]. However, peaks of $\mathrm{LO}_{1}(\mathrm{CdTe}), \mathrm{TO}_{1}(\mathrm{CdTe}), \mathrm{TO}_{2}(\mathrm{ZnTe})$ modes were not observed in the CZT5 sample spectrum, which could be caused by overlapping with an intensive peak of $\mathrm{E}_{T O}(\mathrm{Te})$ mode. The spectrum of CZT6 sample includes weak Te-related $\mathrm{A}_{1}(\mathrm{Te})$ peak, peaks of $\mathrm{TO}_{2}(\mathrm{ZnTe})$ and $\mathrm{LO}_{2}(\mathrm{ZnTe})$ modes, as well as a combination of $\mathrm{LO}_{1}(\mathrm{CdTe})$ and $\mathrm{TO}_{1}(\mathrm{CdTe})$ modes. More- 


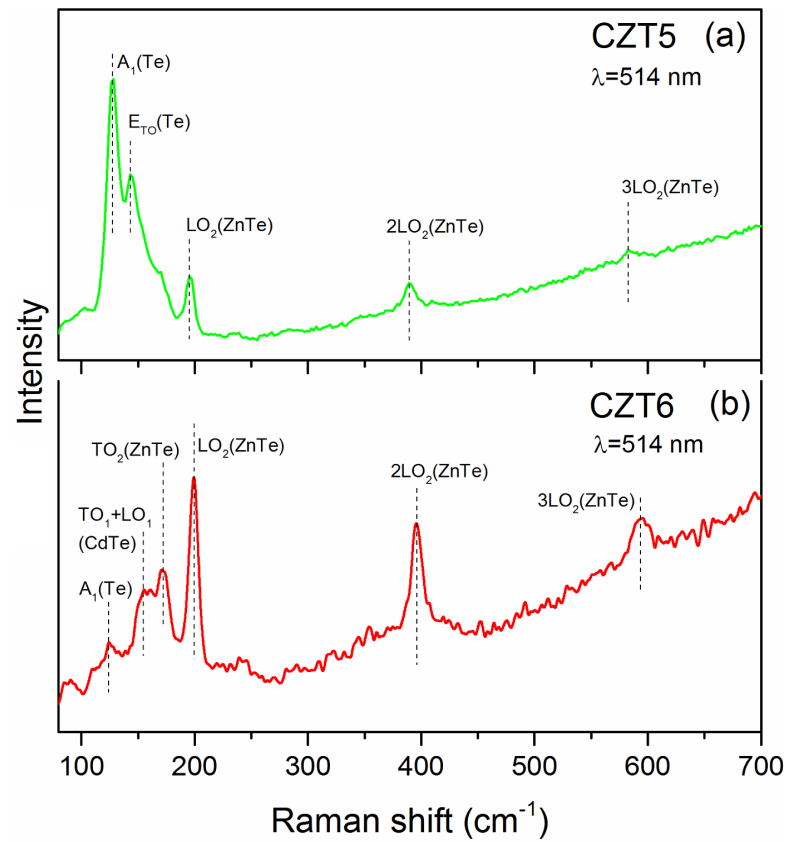

Fig. 5. Room temperature Raman spectra of the CZT5 $(x=0.63)$ and CZT6 $(x=0.68)$ films, measured with $514 \mathrm{~nm}$ excitation.

over, analogously to CZT5 sample, the resonant overtones of $\mathrm{LO}_{2}(\mathrm{ZnTe})$ mode were observed on the spectrum of CZT6 sample.

As it is seen from Fig. 5, measuring of the Raman spectra from CZT5 and CZT6 samples with green laser excitation, which has higher quantum energy than the red laser, led to the appearance of the third-order vibrational modes due to the resonant enhancement of the Raman signal. Thus, it could be considered that green laser excitation is more suitable for measuring of the Raman spectra of Zn-rich $(x>0.60)$ CZT films, than red laser excitation. On the other hand, the application of highenergy green laser led to overheating of the surface of the sample, evaporation of $\mathrm{Cd}$ atoms from the surface and, as a result, to Te-enrichment of the surface. The presence of Te-related modes in the Raman spectra makes it difficult to identify CdTe- and ZnTe-related modes. Therefore in order to avoid overheating of the surface during measuring of the Raman spectra it is necessary to decrease the power of laser excitation.

\section{Conclusions}

In this work, we carried out the study of the Raman spectra, surface morphology, structural and microstructural properties of thick polycrystalline $\mathrm{Cd}_{1-x} \mathrm{Zn}_{x} \mathrm{Te}$ films with $x$ ranged from 0.06 to 0.68 .

The structural analysis showed that the films have zinc-blende crystal structure; the crystal quality vs. Zn concentration relationship has generally accepted nonlinear character, the minimum of this relationship was observed for films with $x$ ranged from 0.46 to 0.63 .
The Raman investigations were carried out using lasers with different wavelength, namely 785, 633, and $514 \mathrm{~nm}$. For CZT films with different zinc concentration the value of excitation wavelength was adjusted in order to achieve high signal-to-noise ratio of the Raman spectra due to resonant enhancement of phonon modes intensities. The Raman spectra had a typical for CZT solid solution twomode behaviour and included CdTe- and ZnTe-like LO and TO modes. In the result of the Raman studies we obtained the dependence of phonon mode frequencies on $x$ for polycrystalline CZT films. The obtained dependence is well correlated with the commonly accepted theory of optical phonons in CZT solid solution. However, we observed the difference between the frequencies of phonon modes in polycrystalline CZT films and CZT single crystals. This difference could be caused by the influence of microstrains in polycrystalline CZT films. However, in general, phonon modes frequencies vs. zinc concentration dependences for CZT single crystal are able to be used as a reference data for identification of phonon modes in the Raman spectra of CZT polycrystalline films.

\section{Acknowledgments}

This work was supported by the Ministry of Education and Science of Ukraine (Grant No. 0115U000665c, 0116U002619), and by Latvian National Research Programme in Materials Science (IMIS2) (2014-2017).

\section{References}

[1] R. Triboulet, P. Siffert, CdTe and Related Compounds; Physics, Defects, Hetero- and Nanostructures, Crystal Growth, Surfaces and Applications, Elsevier, Amsterdam 2010.

[2] M. Fiederle, T. Feltgen, J. Meinhardt, M. Rogalla, K.W. Benz, J. Cryst. Growth 197, 635 (1999).

[3] S. del Sordo, L. Abbene, E. Caroli, A.M. Mancini, A. Zappettini, P. Ubertini, Sensors 9, 3491 (2009).

[4] P. Mahawela, G. Sivaraman, S. Jeedigunta, J. Gaduputi, M. Ramalingam, S. Subramanian, S. Vakkalanka, C. Ferekides, D.L. Morel, Mater. Sci. Eng. B Solid-State Mater. Adv. Technol. 116, 283 (2005).

[5] P.J. Sellin, Nucl. Instrum. Methods Phys. Res. A 563, 1 (2006).

[6] H. Zhou, D. Zeng, S. Pan, Nucl. Instrum. Methods Phys. Res. A 698, 81 (2013).

[7] R. Dhere, T. Gessert, J. Zhou, S. Asher, J. Pankow, H. Moutinho, Mater. Res. Soc. Symp. Proc. 763, 409 (2003).

[8] R.G. Solanki, Indian J. Pure Appl. Phys. 48, 133 (2010).

[9] N. Amin, A. Yamada, M. Konagai, Jpn. J. Appl. Phys. Part 1 41, 2834 (2002).

[10] K. Prabakar, S. Venkatachalam, Y.L. Jeyachandran, S.K. Narayandass, D. Mangalaraj, Mater. Sci. Eng. B 107, 99 (2004). 
[11] J. Tao, H. Xu, Y. Zhang, H. Ji, R. Xu, J. Huang, J. Zhang, X. Liang, K. Tang, L. Wang, Appl. Surf. Sci. 388, 180 (2016).

[12] V. Kosyak, Y. Znamenshchykov, A. Čerškus, Y.P. Gnatenko, L. Grase, J. Vecstaudza, A. Medvids, A. Opanasyuk, G. Mezinskis, J. Alloys Comp. 682, $543(2016)$.

[13] D. Kurbatov, A. Opanasyuk, H. Khlyap, Phys. Status Solidi A 206, 1549 (2009).

[14] C.J. Panchal, A.S. Opanasyuk, V.V. Kosyak, M.S. Desai, I.Y. Protsenko, J. Nano- Electron. Phys. 3, 274 (2011).

[15] S. Perkowitz, L.S. Kim, Z.C. Feng, Phys. Rev. B 42 1455 (1990)

[16] D.N. Talwar, Z.C. Feng, P. Becla, Phys. Rev. B 48, 17064 (1993)

[17] D.J. Olego, P.M. Raccah, J.P. Faurie, Phys. Rev. B 33, 3819 (1986).

[18] M. Jain, II-VI Semiconductor Compounds, World Sci., 1993.

[19] F. Cerdeira, C.J. Buchenauer, F.H. Pollak, M. Cardona, Phys. Rev. B 5, 580 (1972).

[20] D. Nam, H. Cheong, A.S. Opanasyuk, P.V. Koval, V.V. Kosyak, P.M. Fochuk, Phys. Status Solidi C 4 1 (2014).

[21] A.S. Opanasyuk, D.I. Kurbatov, V.V. Kosyak, S.I. Kshniakina, S.N. Danilchenko, Crystallogr. Rep. 57, 927 (2012).

[22] JCPDS, International Centre for Diffraction Data, USA, Card Number 15-0770.

[23] B.E. Warren, X-ray Diffraction, Dover, New York 1990.
[24] Y.V. Znamenshchykov, V.V. Kosyak, A.S. Opanasyuk, E. Dauksta, A.A. Ponomarov, A.V. Romanenko, A.S. Stanislavov, A. Medvids, I.O. Shpetnyi, Yu.I. Gorobets, Mater. Sci. Semicond. Process. 63, 64 (2017).

[25] S. Stolyarova, F. Edelman, A. Chack, A. Berner, P. Werner, N. Zakharov, M. Vytrykhivsky, R. Beserman, R. Weil, Y. Nemirovsky, J. Phys. D Appl. Phys. 41, 65402 (2008).

[26] Y.V. Znamenshchykov, V.V. Kosyak, A.S. Opanasyuk, M.M. Kolesnyk, P.M. Fochuk, Funct. Mater. 23, 1 (2016).

[27] D. Kurbatov, V. Kosyak, M. Kolesnyk, A. Opanasyuk, S. Danilchenko, Integr. Ferroelectr. 103, 32 (2008).

[28] A.R. Bushroa, R.G. Rahbari, H.H. Masjuki, M.R. Muhamad, Vacuum 86, 1107 (2012).

[29] V. Kosyak, Y. Znamenshchykov, A. Čerškus, L. Grase, Y.P. Gnatenko, A. Medvids, A. Opanasyuk, G. Mezinskis, J. Lumin. 171, 176 (2016).

[30] J.L. Reno, E.D. Jones, Phys. Rev. B 45, 1440 (1992).

[31] A. Aydinli, A. Compaan, G. Contreras-Puente, A. Mason, Solid State Commun. 80, 465 (1991).

[32] K.R. Murali, Sol. Energy 82, 220 (2008).

[33] Y.L. Wu, Y.-T. Chen, Z.C. Feng, J.-F. Lee, P. Becla, W. Lu, Hard X-Ray, Gamma-Ray, Neutron Detect. Phys. XI 7449, 74490Q (2009).

[34] S.A. Hawkins, E. Villa-Aleman, M.C. Duff, D.B. Hunter, A. Burger, M. Groza, V. Buliga, D.R. Blacket, J. Electron. Mater. 37, 1438 (2008).

[35] J.F. Scott, R.C. Leite, T.C. Damen, Phys. Rev. 188 1285 (1969). 